\title{
Recurrence of Macular Hole Retinal Detachment after Intravitreal Ranibizumab Injection for the Treatment of Choroidal Neovascularization from the Remaining Macular Hole Edge
}

\author{
Keiko Otsuka ${ }^{a}$ Hisanori Imai $i^{a, b}$ Tsuyoshi Shimoyama ${ }^{a}$ \\ Takayuki Nagai $^{\mathrm{b}}$ Shigeru Honda ${ }^{\mathrm{b}}$ Atsushi Azumi ${ }^{\mathrm{a}, \mathrm{b}}$ \\ ${ }^{a}$ Department of Ophthalmology, Kobe Kaisei Hospital, and ${ }^{b}$ Department of \\ Organ Therapeutics, Division of Ophthalmology, Kobe University Graduate \\ School of Medicine, Kobe, Japan
}

\section{Key Words}

Macular hole - Retinal detachment - Intravitreal ranibizumab injection - Anti-VEGF . Lucentis · Choroidal neovascularization

\begin{abstract}
Purpose: To report a case who had recurrence of macular hole retinal detachment (MHRD) after intravitreal ranibizumab injection (IVR) for the treatment of choroidal neovascularization (CNV) that arose from the damaged retinal pigment epithelium of the remaining macular hole $(\mathrm{MH})$ edge, which had been successfully treated by pars plana vitrectomy (PPV) 15 years previously.

Case Report: A 67-year-old man with previous PPV for MHRD secondary to high myopia in the right eye had been under observation for 15 years after surgery. The retina had been successfully attached, but the MH remained open. He had CNV which arose from the remaining $\mathrm{MH}$ edge. IVR was performed for the treatment of $\mathrm{CNV}$. One month after the injection, CNV was contracted but recurrence of MHRD occurred. PPV with an additional internal limiting membrane peeling, removal of the CNV membrane and 20\% SF6 gas tamponade was performed. One year after the last surgery, his right retina was attached and the $\mathrm{MH}$ was closed successfully.

Conclusion: We propose that patients who undergo IVR should be carefully maintained and followed up for possible complications including the recurrence of MHRD.
\end{abstract}




\section{Introduction}

Intravitreal anti-vascular endothelial growth factor (VEGF) agent therapy for agerelated macular degeneration (AMD), proliferative diabetic retinopathy (PDR) and neovascular glaucoma (NVG) is widely used. On the other hand, retinal pigment epithelium (RPE) tears [1-3] and worsening of tractional retinal detachment (RD) [46] have been reported as a result of the contractile forces of regressing neovascular membranes after intravitreal anti-VEGF agent injection. However, the mechanism of construction of the fibrovascular membranes remains to be elucidated.

In this report, we present a case who had recurrence of macular hole retinal detachment (MHRD) after intravitreal ranibizumab injection (IVR) for the treatment of choroidal neovascularization (CNV) that arose from the damaged RPE of the remaining macular hole (MH) edge.

\section{Case Report}

A 67-year-old man with previous pars plana vitrectomy (PPV) for MHRD secondary to high myopia in the right eye (OD) had been under observation for 15 years after the surgery. Slit lamp examination revealed pseudophakia in OD and mature cataract in the left eye (OS). Funduscopic finding revealed attached retina and remaining $\mathrm{MH}$ in OD. The fundus condition in OS was invisible due to the mature cataract. Best corrected Landolt ring chart visual acuity (BCVA) was from 0.1 to 0.2 in OD and $30 \mathrm{~cm}$ finger motion in OS. The patient presented with sudden vision loss in OD. On presentation, his BCVA was 0.1 . Funduscopic examination revealed a disciform RPE atrophy formation at the bottom of the remaining $\mathrm{MH}$ and $\mathrm{CNV}$ development around the edge of $\mathrm{MH}$ from the superior to temporal side (fig. 1a, d, f). His BVCA was not worsening but his subjective symptom was getting worse day by day. IVR was performed after 14 days from the onset for the treatment of CNV. One month after the injection, he presented with visual disturbance in OD. Funduscopic examination showed CNV contraction to the superior edge and recurrence of MHRD from the inferior edge of the remaining $\mathrm{MH}$ (fig. 1b, g). Emergency PPV with an additional internal limiting membrane peeling, removal of CNV and 20\% SF6 gas tamponade was performed on the same day. Extraction of CNV was performed under the patient's written informed consent, the approval of the institutional review board in Kobe Kaisei Hospital and the Tenets of the Declaration of Helsinki. One year after the last surgery, his right retina was reattached and MH was closed successfully (fig. 1c, e, h). His BVCA was improved at 0.3.

\section{Comment}

RPE tears and worsening of tractional RD after intravitreal anti-VEGF agent therapy have been reported [1-6]. Several reports hypothesized that intravitreal anti-VEGF agent therapy may cause severe contract force of the neovascular membrane followed by RPE tear in AMD [1-3] and tractional RD in PDR [4-6]. It is possible that the contract force of the regressing CNV might be increased after IVR, thereby causing RD from the remaining $\mathrm{MH}$ edge in our case.

In our case, we performed emergency PPV with CNV removal to fix the detached retina because the visual acuity in OS was bad and the MHRD in OD was expanding. For the treatment of CNV, it might be one of the options to continue IVR because IVR is thought less invasive compared with CNV removal during PPV. But if we perform additional IVR first, it is very important to make the CNV inactive before the following PPV because IVR itself may cause recurrence of MHRD due to the contract force of the CNV again. We must follow-up this patient for at least another month after the injection 
to confirm the effect of IVR. Unfortunately, we had not a moment to lose in this case. We performed PPV with CNV extraction and achieved both retinal reattachment and the quieting effect of the CNV.

In summary, we propose that patients who undergo IVR should be carefully maintained and followed up for possible complications including the recurrence of MHRD.
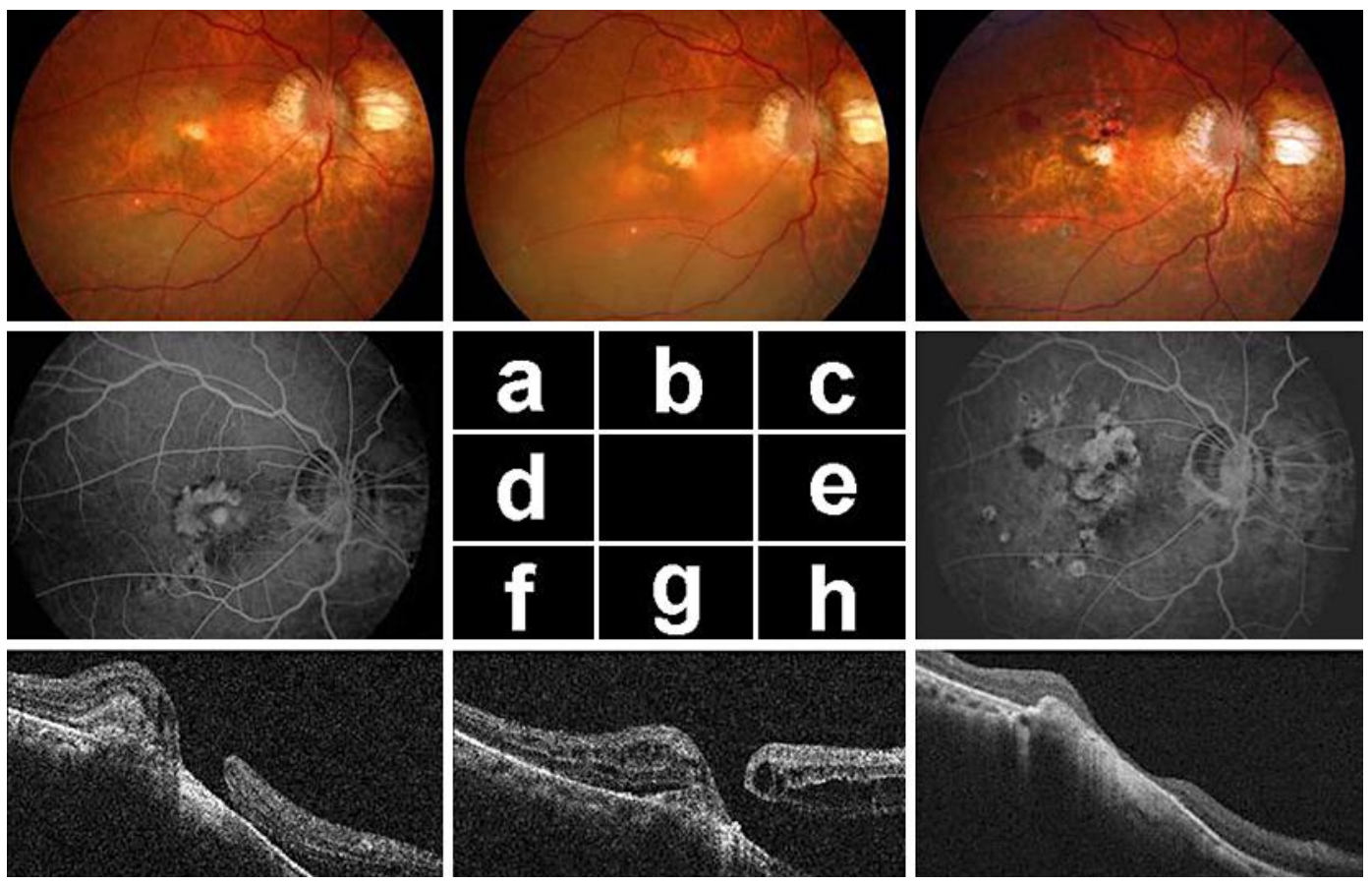

Fig. 1. Before IVR, fundus photograph suggested CNV development from the remaining MH edge (a). FA demonstrated leakage from CNV (d). OCT depicted subretinal CNV (f). One week after IVR, fundus photograph (b) and OCT (g) showed contraction of CNV and recurrence of MHRD. One year after PPV, retina was reattached and MH was closed successfully (c). OCT showed MH closure and retina reattachment (h). CNV removal and chorioretinal atrophy after PPV was confirmed by FA (e). 


\section{References}

1 Gutfleisch M, Heimes B, Schumacher M, Dietzel M, Lommatzsch A, Bird A, Pauleikhoff D: Long-term visual outcome of pigment epithelial tears in association with anti-VEGF therapy of pigment epithelial detachment in AMD. Eye 2011;25:1181-1186.

-2 Chan CK, Lin SG: Retinal pigment epithelial tear after ranibizumab therapy for subfoveal fibrovascular pigment epithelial detachment. Eur J Ophthalmol 2007;17:674-676.

-3 Ronan SM, Yoganathan P, Chien FY, Corcóstegui IA, Blumenkranz MS, Deramo VA, Elner SG, Fastenberg DA, Johnson MW, López M, Mateo C, Moshfeghi DM, Navarro R, Rosenblatt BJ, Sanislo SR, Saxe SJ, Zacks DN: Retinal pigment epithelium tears after intravitreal injection of bevacizumab (avastin) for neovascular age-related macular degeneration. Retina 2007;27:535-540.

\4 Arevalo JF, Maia M, Flynn HW Jr, Saravia M, Avery RL, Wu L, Eid Farah M, Pieramici DJ, Berrocal MH, Sanchez JG: Tractional retinal detachment following intravitreal bevacizumab (Avastin) in patients with severe proliferative diabetic retinopathy. Br J Ophthalmol 2008;92:213-216.

-5 Jonas JB, Schmidbauer M, Rensch F: Progression of tractional retinal detachment following intravitreal bevacizumab. Acta Ophthalmol 2009;87:571-572.

-6 Tranos P, Gemenetzi M, Papandroudis A, Chrisafis C, Papadakos D: Progression of diabetic tractional retinal detachment following single injection of intravitreal Avastin. Eye 2008;22:862. 